\title{
Predicting Credentialed Evaluator Status: Characteristics, Comparisons, and Implications for the CE Program
}

\author{
Karen Lawson \\ University of Saskatchewan \\ Theresa Hunter and Jim McDavid \\ University of Victoria
}

\begin{abstract}
The CES membership database was analyzed in order to determine the socio-demographic profile that best defines Credentialed Evaluators (CE). In general, those currently holding CE status tend to be employed in the private sector, have a $\mathrm{PhD}$-level education, have long-term experience in evaluation, have a major focus on evaluation within their professional activities, and be located within the Atlantic region, Western Canada, or the National Capital Chapter region. Implications of these trends for the sustainability of the CE designation program and options for broadening the scope of uptake within the CES membership are discussed.
\end{abstract}

Keywords: credentialed evaluator, evaluation practice, professional designation, professionalization

Résumé : Une analyse de la base de données des membres de la SCE a été effectuée afin de dégager le profil sociodémographique des évaluateurs qualifiés (EQ). Dans l'ensemble, les actuels détenteurs du titre d'EQ ont tendance à : travailler dans le secteur privé; avoir étudié au niveau du doctorat; posséder une vaste expérience dans le domaine; concentrer principalement leurs activités professionnelles sur l'évaluation; et résider dans les régions de l'Atlantique et de l'Ouest canadien, ainsi que dans la région de la capitale nationale (Ottawa). L'article discute des répercussions de ces tendances sur la pérennité du programme de désignation d'EQ ainsi que des options qui peuvent être envisagées pour encourager l'adhésion d'évaluateurs et d'évaluatrices à la SCE.

Mots clés : évaluateur qualifié, évaluatrice qualifiée, pratique de l'évaluation, désignation professionnelle, professionnalisation

The purpose of this paper is to compare members of the Canadian Evaluation Society who have earned a Credentialed Evaluator (CE) designation to those who have not. The main question that we will address in this paper is whether there

Corresponding author: Karen Lawson, Department of Psychology, University of Saskatchewan, 9 Campus Drive, Saskatoon, SK, S7N 5A5; karen.lawson@usask.ca 
are significant socio-demographic and experiential differences between those who have the CE designation and those who do not. A second question that follows is whether the profile of those who are successful in earning the CE designation has implications for the Canadian Evaluation Society and for the future of the professional designation program.

\section{BACKGROUND}

Since the early 1970s, an increasing demand for evaluation services combined with a dearth of trained, competent evaluators created a situation in which many evaluations were being conducted by individuals ill-equipped for such projects (Love, 2015; Montrosse-Moorhead, Bellara, \& Gambino, 2017; Schwandt, 2015). This inevitably led to evaluations that were poorly designed and conducted, rendering them irrelevant to the evaluation sponsor (Gussman, 2005; Love, 2015) and costly to the sponsor, society, and the reputation of the evaluation field. Professionalization of evaluation has long been debated as a potential means of addressing this issue (for in-depth discussions of issues surrounding professionalization of evaluation please see Altschuld, 2005; Altschuld \& Engle, 2015b; Buchanan, 2015; Castro, Fragapane, \& Rinaldi, 2016; Furubo \& Stame, 2019; Jacob \& Boisvert, 2010; Julnes \& Bustelo, 2017; Long, 2006; Love, 1994, 2015; Montrosse-Moorhead et al., 2017).

As a step toward professionalization, in 2009 the Canadian Evaluation Society (CES) established the Credentialed Evaluator (CE) designation program for its members. At the time, CES was one of two national evaluation societies to offer members a way to distinguish their practice in this way, the other being the Japan Evaluation Society (Buchanan \& Kuji-Shikatani, 2013). For the individual evaluator, CES promotes the CE designation as a means of conveying evaluation competence, of demonstrating a commitment to the profession, of guiding professional development, and of obtaining a competitive edge in evaluation-related practice fields. At the broader level, the $\mathrm{CE}$ designation is designed to enhance the quality of evaluation practice in Canada and the professionalization of evaluation as a distinct field (Buchanan, 2015; Love, 2015).

Earning CE status involves a process whereby members apply to have their achievements and professional experience adjudicated by a peer review committee and formally recognized as meeting the criteria necessary for being designated a competent evaluator. This designation, as a credential, is awarded on the basis of having satisfied or completed specific educational and experiential criteria and is distinguished from either certification or a licensure, which are awarded, respectively, following independent verification that the applicant has mastered specified competencies or skills, usually through post-secondary educational attainment and examinations or, in the case of licensure, satisfying certification requirements, as well as legal requirements to practice (Altschuld, 2005; Altschuld \& Engle, 2015a; Halpern, Gauthier, \& McDavid, 2015).

The three criteria specified in the Credentialed Evaluator information "For candidates" (CES, n.d. a) for successful CE candidates are the following: 
1. Evidence of a graduate degree [there is no indication that this degree needs to be evaluation-related] OR a graduate certificate/diploma specifically in Program Evaluation from a recognized university.

2. Two years of full-time equivalent evaluation-related work experience within the last 10 years. Letters of reference are required to substantiate the statements of work experience.

3. A combination of education and/or work experience that covers $70 \%$ of the competencies in each of the five domains of professional practice: reflective practice, technical practice, situational practice, management practice, and interpersonal practice. Applicants outline how their educational and/or professional experiences reflect each targeted competency within a short narrative not exceeding 150 words.

\section{Application and maintenance process}

The CE application process is presented on the CES website under the heading Credentialed Evaluator information "For candidates" (CES, n.d. a) and is summarized by Kuji-Shikatani, Thompson, and Matthew (2015). As outlined by these sources, a CES member initiates the process by registering online and providing the application fee of $\$ 485$ (this is a one-time fee that covers the administrative costs associated with application review). Following this, the applicant is granted access to the online CE application site. The entire application is completed online (in either French or English), and the applicant is allowed 36 months to complete the submission. Once submitted, the applications are anonymously reviewed by two members of the CES Credentialing Board. A third reviewer will review the file in the event that the initial reviewers disagree. Unsuccessful applicants will be given feedback regarding how to strengthen their submission in order to better meet the requirements.

Although this is the standard route to pursue the professional designation, certain CES members have achieved the credential via two alternative paths. First, in order to form the initial Credentialing Board, the CES President offered all CES Fellows and award winners an honorary CE along with an invitation to join the Board, an offer that more than a dozen individuals accepted (Kuji-Shikatani et al., 2015). Second, in order to address low initial uptake rates and create a critical mass of individuals with the designation, CES implemented a temporary one-year "fast-track" application process in 2012 to facilitate the applications of CES members whose experiences met or exceeded the CE requirements. Specifically, this expedited application process was made available to those who held a CES membership for at least seven years, had graduate-level education, and performed evaluation functions as a primary or major part of their employment (Kuji-Shikatani, 2015; Kuji-Shikatani et al., 2015). The streamlined applications were reviewed by the Credentialing Board on the basis of the same criteria as those applying through the regular route.

Once obtained, CE status must be continually maintained via well-laid-out requirements. Specifically, in addition to a $\$ 50$ annual maintenance fee, it must be 
renewed every three years by demonstrating 40 hours of professional development across diverse eligible categories, including participating in workshops, teaching courses, publishing, and engaging in CES organizational duties, to name a few (CES, n.d. b; Kuji-Shikatani et al., 2015). The annual fee is paid alongside the annual CES membership fee, and the professional development hours are logged online on the CE's member account.

\section{Benefits and uptake of CE status}

Many commentators have noted that a lack of a coherent evaluation identity is the most serious obstacle facing efforts to professionalize evaluation (Castro et al., 2016; Mason \& Hunt, 2018; Montrosse-Moorhead et al., 2017; Picciotto, 2011). As discussed by Mason and Hunt (2018), and framed within the tenets of social identity theory (Tajfel \& Turner, 2004), group identification carries many benefits. Specifically, individuals who identify with a social group are more likely to act in ways consistent with the group identity, cooperate with other group members, and internalize the values of the larger group. The CE designation offered by CES can potentially be a means of promoting such group identity and advancing the field within the diverse Canadian evaluation landscape. In fact, for the individual evaluator, CES promotes the CE designation as a means not only of conveying evaluation competence and obtaining a competitive edge in evaluation-related practice fields but also of demonstrating a commitment to the profession and guiding professional development. As preliminary evidence that the CE designation is making strides in creating a group identity among evaluators, in response to a recent survey of CES members (Gauthier, Kishchuk, Borys, \& Roy, 2015), CE holders were significantly more likely than non-holders to feel that they "belong to a recognized profession." In line with social identity theory (Tajfel \& Turner, 2004), these CE holders were also more likely to align their evaluation practices with the stated CES competencies, potentially evidencing an internalization of, and/or a desire to act in accordance with, the values of the larger group.

Although the potential benefits of the CE designation for evaluators are clearly defined, past examinations of uptake rates reveal that only a minority of CES members (14-16\%) hold the designation (Gauthier et al., 2015; KujiShukatani, 2015). Further, these examinations provide evidence that the uptake of the credential is not equal across the membership. Specifically, compared to CES members without the credential, CE holders were found to be older, more experienced in evaluation, more likely to work in the private sector, and less likely to be located in Quebec and Ontario (Gauthier et al., 2015). Evaluator age and experience are highly correlated, and, at the time of their survey in 2014, Gauthier et al. (2015) found that the highest uptake rate was evident among the most experienced evaluators (those with 11 or more years in the field), a finding that they partially attribute to the temporary fast-track option that specifically targeted experienced evaluators. The authors further posit that the preponderance of federal government employees within the CES membership in central Canada underlies to a large extent the regional distribution of CEs. But the study also reports a lack 
of recognition of the $\mathrm{CE}$ status for evaluators within the federal government that diminishes the perceived benefits to the individual, likely resulting in a lower CE uptake rate in that group. This finding is reflected in a recent critical assessment of the CE program (Adrien \& Hellrung, 2019). In a related vein, the perceived benefits are likely highest for those in the private sector, resulting in a heightened uptake rate.

A recent evaluation of the Credentialed Evaluator Designation Program concluded that although the program is making great strides, many Canadian evaluation practitioners and commissioners are still uncertain about the overall benefits or relevance of the designation (Fierro, Galport, Hunt, Codd, \& Donaldson, 2016). In 2014, dubious benefits combined with the associated financial costs (i.e., the application fee) and the time-intensive nature of completing the application appeared to deter many from pursuing CE status (Gauthier et al., 2015). Given the voluntary nature of the Designation Program (those who do not hold CE status are in no way limited or curtailed in their ability to practise, again in contrast to professions in which certification or licensure is necessary in order to practise [Altschuld, 2005; Altschuld \& Engle, 2015a; Halpern et al., 2015]) and the growing global movement toward evaluation professionalization (e.g., Castro et al., 2016; Furubo \& Stame, 2019; Julnes \& Bustelo, 2017; McDavid \& Huse, 2015; Montrosse-Moorhead et al., 2017), it is worthwhile to continually monitor uptake patterns by comparing CES members who hold the CE designation to those who do not.

\section{METHODOLOGY}

The analyses in this paper are based on an anonymized database reflecting the complete CES membership as of May 2017. This database contains the following basic demographic information on CES members: CE status, chapter affiliation, age, gender, preferred language, highest level of education, education, employment classification, degree of focus on evaluation within current employment, and length of time in the evaluation field. In total, 1,741 CES members were contained within the database. A minority of the total membership $(n=306 ; 17.6 \%)$ held CE status. See Table 1 for a summary of the socio-demographic profile of CES members.

CE status (yes or no) was coded by CES administrators prior to the deidentification of the database, so information on this variable was complete. However, as the majority of the database is populated via the demographic information provided by members on their membership profile, there was an extensive amount of missing data on the other variables. In order to maximize the uses of the information available, bivariate chi-square analyses comparing the CE holders with non-CE holders on relevant socio-demographic variables were conducted with the portion of the database that was complete for the individual variable in question, resulting in different sample sizes for each analysis.

Evaluators are aware of the importance of examining the correlates of salient group membership during the evaluation process. Frequencies, group averages, 
Table 1. Socio-demographic profile of CES members

\begin{tabular}{|c|c|c|}
\hline Demographic variables & $n$ & $\%$ \\
\hline CE & 306 & $17.6 \%$ \\
\hline Non-CE & 1,435 & $82.4 \%$ \\
\hline \multicolumn{3}{|l|}{ Age } \\
\hline 35 and under & 449 & $25.8 \%$ \\
\hline $36-50$ & 534 & $30.7 \%$ \\
\hline Over 50 & 450 & $25.8 \%$ \\
\hline Missing data & 308 & $17.7 \%$ \\
\hline \multicolumn{3}{|l|}{ Chapter affiliation } \\
\hline National Capital & 371 & $21.3 \%$ \\
\hline Western & 587 & $33.7 \%$ \\
\hline Atlantic & 116 & $6.7 \%$ \\
\hline Eastern & 557 & $32.0 \%$ \\
\hline Other & 109 & $6.3 \%$ \\
\hline Missing data & 1 & $0.1 \%$ \\
\hline \multicolumn{3}{|l|}{ Education level } \\
\hline BA & 209 & $12.0 \%$ \\
\hline MA & 855 & $49.1 \%$ \\
\hline $\mathrm{PhD}$ & 359 & $20.6 \%$ \\
\hline Missing data & 318 & $18.3 \%$ \\
\hline \multicolumn{3}{|l|}{ Employment type } \\
\hline Provincial government & 271 & $15.6 \%$ \\
\hline Federal government & 213 & $12.2 \%$ \\
\hline Municipal government & 78 & $4.5 \%$ \\
\hline Self-employed & 236 & $13.6 \%$ \\
\hline University/college & 190 & $10.9 \%$ \\
\hline Private business & 165 & $9.5 \%$ \\
\hline Other & 339 & $19.5 \%$ \\
\hline Missing data & 249 & $14.3 \%$ \\
\hline \multicolumn{3}{|l|}{ Focus on evaluation } \\
\hline Primary & 626 & $36.0 \%$ \\
\hline Major & 538 & $30.9 \%$ \\
\hline Minor & 278 & $16.0 \%$ \\
\hline None & 19 & $1.1 \%$ \\
\hline Missing data & 280 & $16.1 \%$ \\
\hline \multicolumn{3}{|l|}{ Time in evaluation } \\
\hline Less than 2 years & 246 & $14.5 \%$ \\
\hline $2-5$ years & 314 & $18.6 \%$ \\
\hline $6-10$ years & 320 & $18.9 \%$ \\
\hline $11-15$ years & 219 & $13.0 \%$ \\
\hline $16-20$ years & 137 & $8.1 \%$ \\
\hline More than 20 years & 228 & $13.5 \%$ \\
\hline Missing data & 227 & $13.4 \%$ \\
\hline
\end{tabular}




\begin{tabular}{lrr}
\hline Demographic variables & \multicolumn{1}{c}{$n$} & $\%$ \\
\hline Gender & & \\
Male & 447 & $25.7 \%$ \\
Female & 1,024 & $58.8 \%$ \\
Missing data & 270 & $15.5 \%$ \\
Preferred language & & \\
French & 106 & $6.1 \%$ \\
English & 1,635 & $93.9 \%$ \\
\hline
\end{tabular}

Note. "Missing data" includes both non-responses and "prefer not to answer" selections by respondents

and chi-square analyses provide descriptions of group membership delineated by salient variables, but they do not address the question of which of these variables are the best predictors of group membership, or of how these variables relate to the odds of achieving specific outcomes.

Multivariate logistic regression was conducted with the portion of the database that was complete for all included variables $(n=1282 ; 252$ CEs and 1030 non-CEs). Logistic regression is a multivariate statistical method to examine predictors of group membership within a dataset and weigh the relative importance of those predictors. The logistic regression in this paper refines the bivariate findings and provides a basis for the conclusions and implications that follow from our analyses.

\section{RESULTS}

\section{Findings from the bivariate analyses}

As an initial step, chi-square tests of association were conducted to determine whether the basic demographic variables are individually associated with CE status. Those variables that were associated with CE status were further explored in the subsequent logistic regression. Prior to the analyses, the demographic variables were recoded into conceptually meaningful response categories to facilitate interpretation.

\section{Member age}

Member age was recoded into three groups: 35 and under, 36-50, and over 50. The chi-square analysis indicated a significant association between member age and CE designation status, $\chi^{2}(2, n=1433)=48.3, p<0.001$. Post-hoc tests revealed that the youngest and oldest groups deviated from the expected frequencies. Fewer members aged 35 and under hold the CE designation than expected $(p<0.001)$, and more members over 50 years old hold the CE designation than 
expected $(p<0.001)$. The middle age grouping did not deviate from the expected distribution. Figure 1 compares the total percentage of CES members within each age grouping with the percentage of those holding the $\mathrm{CE}$ designation within each age grouping. While individuals aged 35 years and under comprise $31 \%$ of the CES membership, they account for only $15 \%$ of CE holders. Alternatively, although those over the age of 50 comprise approximately the same proportion of the CES membership base (31\%), they account for almost one-half (46\%) of the CE holders.

\section{Chapter affiliation}

CES Chapter Affiliation was recoded into five groups: West (BC \& Yukon, Alberta \& NWT, Saskatchewan, Manitoba); East (Ontario [outside of the National Capital Region], SQEP [La Société québécoise dévaluation de programme], National Capital Chapter [Ottawa]); Atlantic (New Brunswick, Nova Scotia, PEI, Newfoundland \& Labrador); and Other (International). Note that the Other category was omitted for the subsequent regression analysis. CE designation status was significantly associated with chapter affiliation, $\chi^{2}(4, n=1740)=28.3, p$ $<0.001$. Post-hoc tests reveal that the East, National Capital Chapter, and Other groups deviated from the expected frequencies. Specifically, the National Capital Chapter contained more CE holders than expected $(p<0.001)$, while both the East $(p=0.002)$ and Other $(p=0.008)$ categories contained fewer than expected. While the National Capital Chapter members comprise just over $20 \%$ of the CES membership, they account for approximately $30 \%$ of those holding the CE designation. On the other hand, although Ontario and SQEP members (East category) account for almost one-third of the CES membership (32\%), they comprise only approximately one-quarter (24.5\%) of CE holders (see Figure 2).

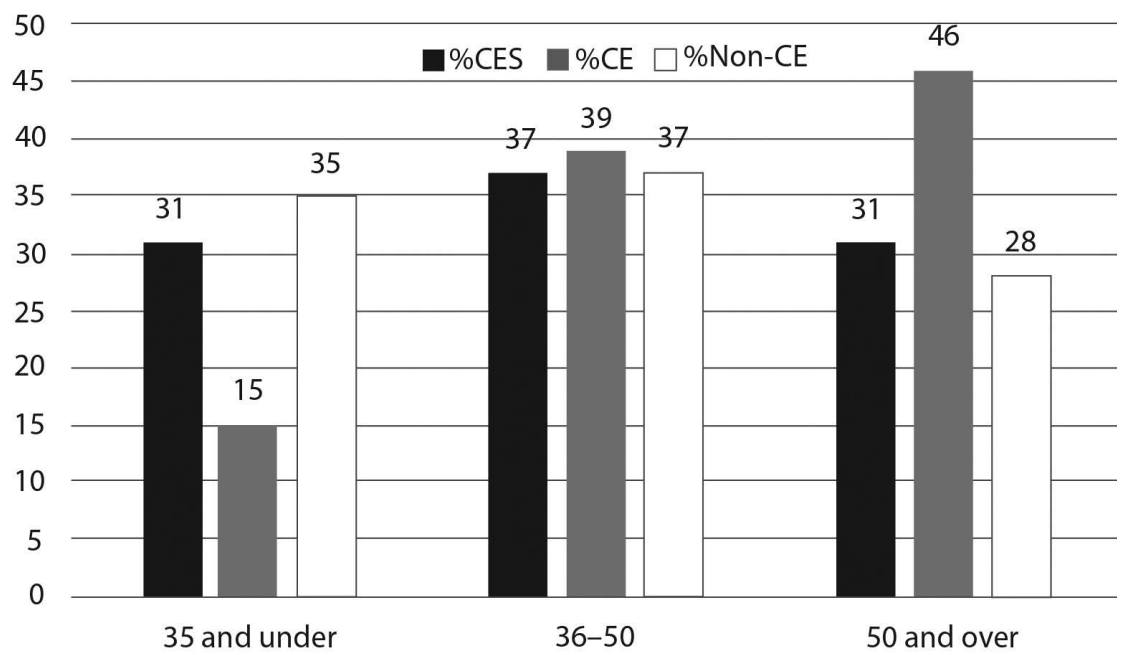

Figure 1. Age: percentages by CE status 


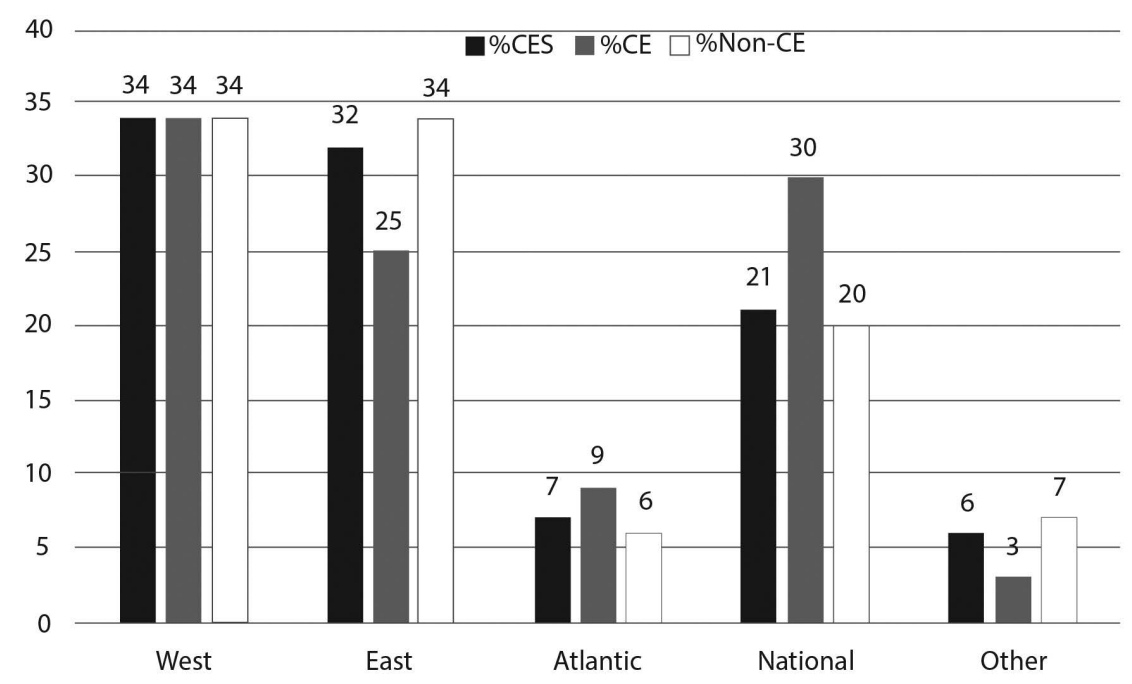

Figure 2. Chapter affiliations: Percentages by CE status

\section{Highest level of education}

Highest level of education (BA, $\mathrm{MA}, \mathrm{PhD}$ ) was significantly associated with $\mathrm{CE}$ status, $\chi^{2}(2, n=1423)=21.3, p<0.001$. Specifically, more PhDs have CE status than would be expected. One-third (33\%) of CEs have a PhD, compared to only one-quarter of the total CES membership base. On the other hand, fewer than expected Bachelor-level CES members hold CE status, but this is likely due to the fact that graduate-level training is normally a requirement for the designation. Figure 3 summarizes the total percentage of CES members within each educational attainment level compared with those holding the CE designation.

\section{Employment classification}

Type of employment was classified into the following categories: Provincial Government, Self-Employed, Federal Government, University/College, Private Business, Municipal Government, and Other (School, Foundation, Retired, Student). CE designation status was significantly associated with type of employment, $\chi^{2}$ $(6, n=1492)=73.9, p<0.001$. Post-hoc tests reveal that CES members who are self-employed $(p<0.001)$ or employed within a private business $(p<0.001)$ are more likely than expected to hold CE status. In contrast, fewer than expected of those employed within a municipal government setting $(p=0.005)$ or who fell within the "Other" employment category $(p=0.0001)$ held the CE designation. No other categories deviated from the expected norm. Almost half $(46.7 \%)$ of those with CE status are either self-employed or employed within the private sector (see Figure 4). Based on these findings, for the purpose of the subsequent regression analysis, this variable was recoded into two groups for conceptual parsimony: Public Sector (Provincial Government, Federal Government, Municipal 


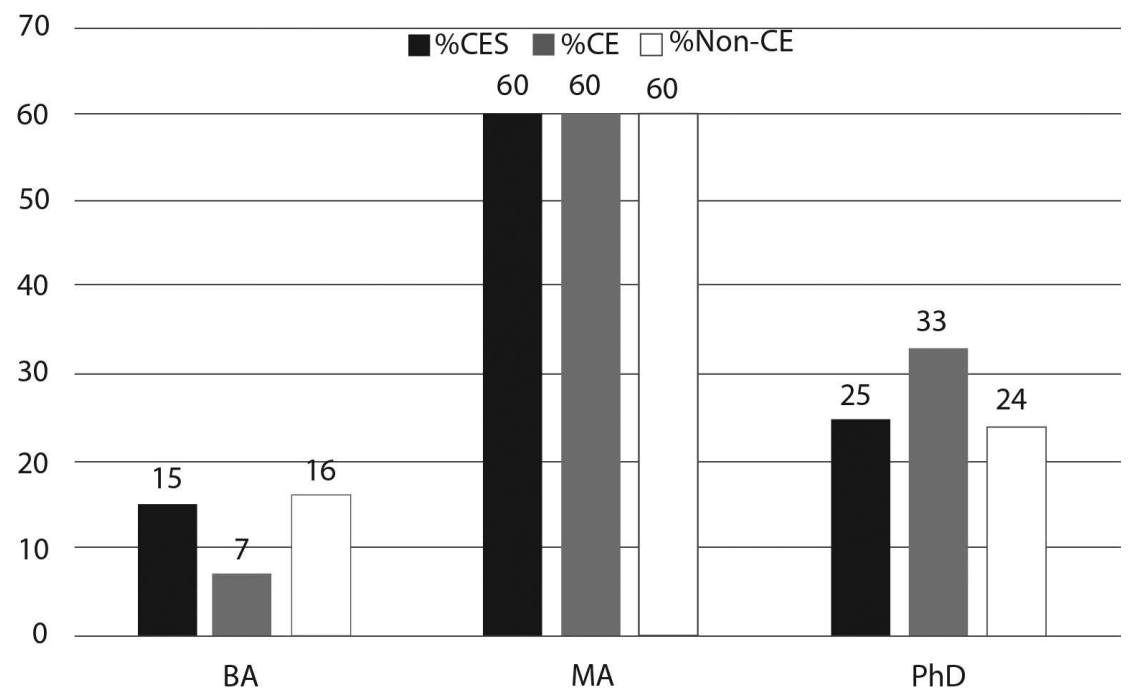

Figure 3. Highest level of education: percentages by CE status

Government, University/College, Other) and Private Sector (Private Business, Self-Employed).

\section{Employment focus on evaluation}

The degree of focus on evaluation within current employment was coded as primary, major, minor, or none. A chi-square test indicated that employment focus on evaluation was significantly associated with CE designation status, $\chi^{2}$ $(3, n=1461)=36.9, p<0.001$. Post-hoc tests revealed that those members with

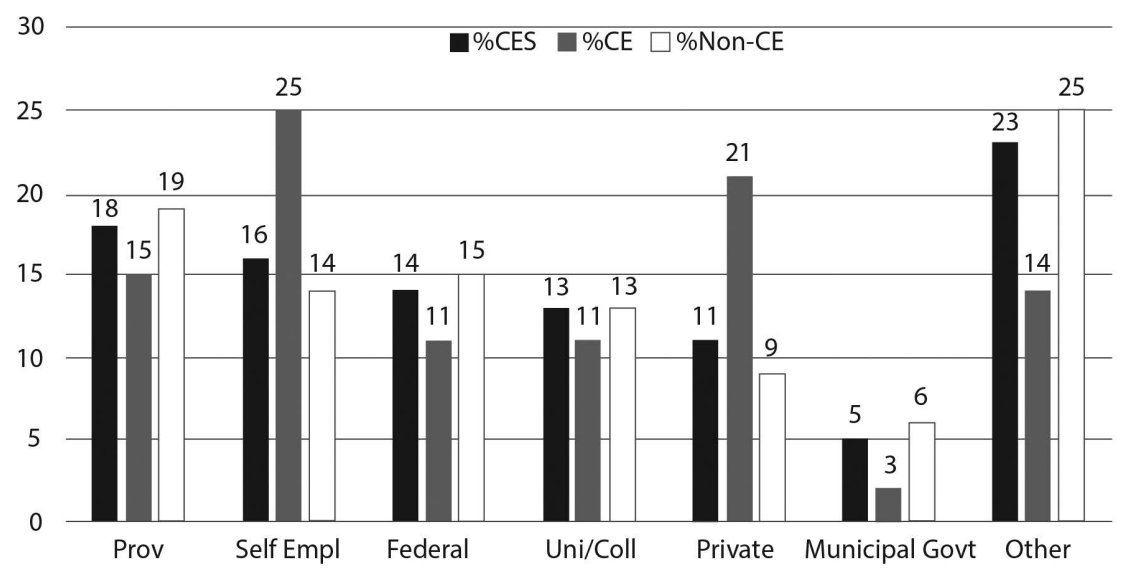

Figure 4. Employment classification: percentages by CE status 


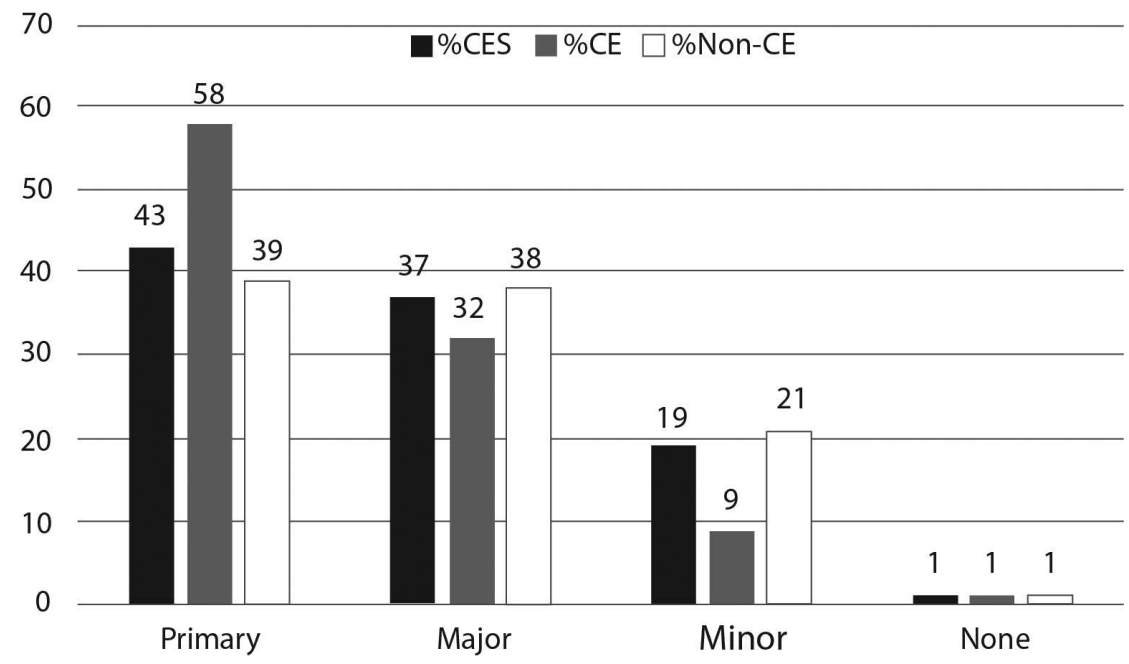

Figure 5. Focus on evaluation: percentages by CE status

a primary focus on evaluation within their employment were more likely than expected to hold the CE designation $(p<0.001)$, while those with a minor focus were less likely than expected $(p<0.001)$. Those who indicated either a major focus or no focus at all on evaluation within their job did not deviate from the expected. In total, almost $60 \%$ of CE holders indicated that their employment involves a primary focus on evaluation (see Figure 5). Note that due to the very small sample size within the "None" category, it was combined with the "Minor" category for the subsequent regression analysis.

\section{Length of time practising evaluation}

This variable was coded into the following categories: less than 2 years, $2-5$ years, 6-10 years, $11-15$ years, $16-20$ years, over 20 years. CE designation was significantly associated with length of time practising evaluation, $\chi^{2}(5, n=1464)=195.5$, $p<0.001$. With the data collapsed into three groupings for conceptual parsimony [early career (5 years or less); mid-career (6-15 years); later career (over 16 years)], post-hoc tests reveal that CES members who are newer to the field of evaluation ( 5 years or less) are less likely to hold the credential $(p<0.001)$, while those in the later stages of their career (practising in the evaluation field for 16 or more years) were more likely than expected to hold the CE designation $(p<$ 0.001). In fact, over half (52\%) of all CE holders report practising in the evaluation field for 16 or more years (see Figure 6).

\section{Gender}

Identified gender was not significantly associated with CE designation status, $\chi^{2}$ $(1, n=1471)=0.075, p=0.78$. The proportion of women and men both with and 


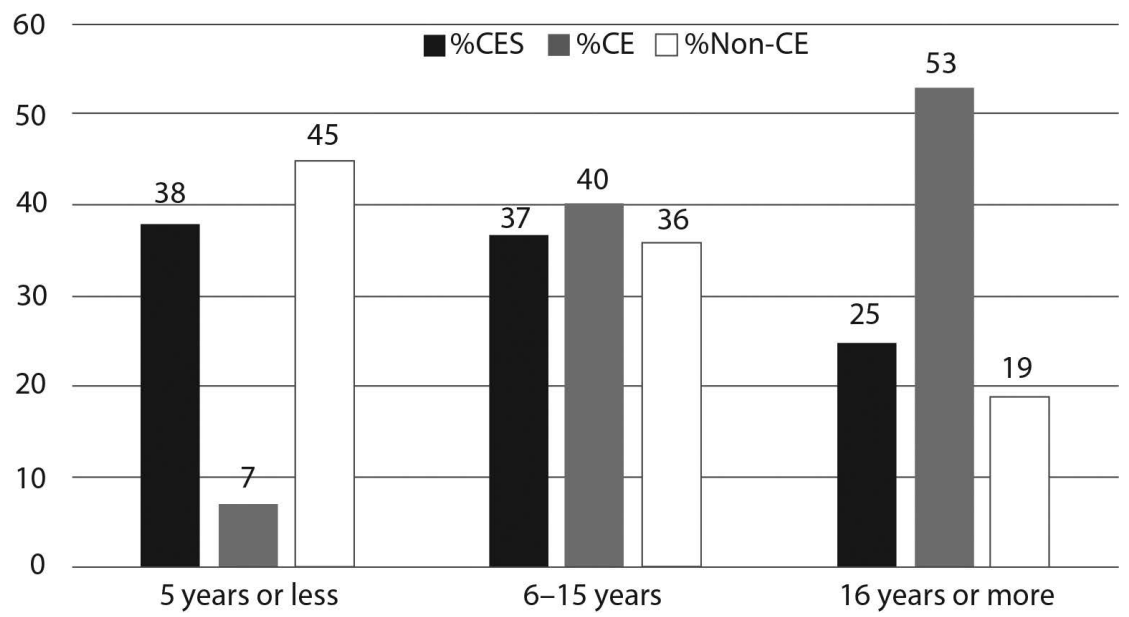

Figure 6. Length of evaluation career: percentages by CE status

without the $\mathrm{CE}$ designation were statistically identical and mirrored the proportions of men and women with CES membership (see Figure 7).

\section{Preferred language}

Preference for French versus English as the primary language of correspondence was not associated with holding the CE designation, $\chi^{2}(1, n=1486)=1.49$, $p=0.22$ (see Figure 8).

\section{Findings from the logistic regression analysis}

Logistic regression is a statistical technique that fits a regression line to data where the outcome (dependent) variable is categorical (Howell, 2010). Unlike related techniques, such as discriminant function analysis, logistic regression makes no assumptions regarding linearity, normality, or the level of the measurement of the predictor variables (i.e., they can be discrete, continuous, or a mix) (Tabachnik \& Fidell, 2013). Given these basic assumptions, it is the analysis of choice when the goal is to predict group membership from a diverse collection of predictor variables. Therefore, we chose logistic regression analysis to determine which socio-demographic characteristics of CES members best predict their CE designation status.

Our outcome variable is dichotomous in that a CES member either holds the $\mathrm{CE}$ designation or does not. The bivariate analyses conducted in the initial phase of our analysis informed our decisions regarding predictor variables to enter into the regression equation. Specifically, only the demographic variables that evidenced a significant association with CE status were included in the subsequent regression analysis. In order to avoid the errors associated with multicollinearity (high correlations among predictor variables; Tabachnik \& Fidell, 2013), member age, even 


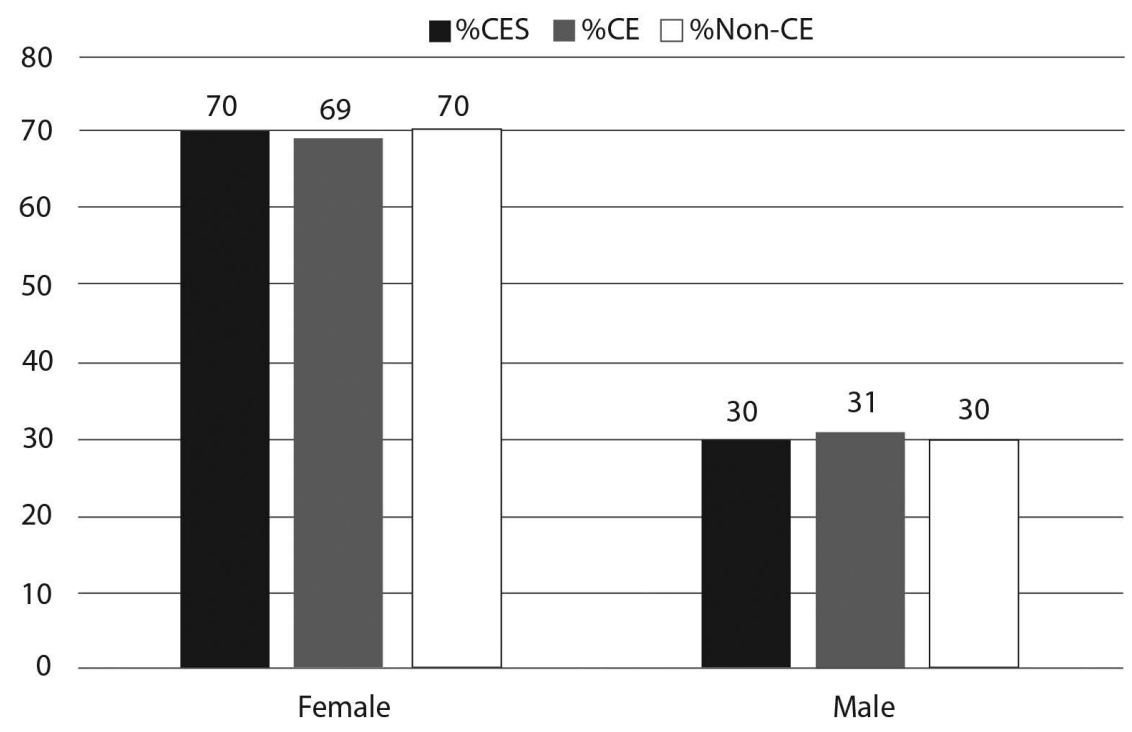

Figure 7. Gender: percentages by CE status

though significantly associated with CE status at the bivariate level, was omitted from the analysis due to its high correlation with time practising evaluation. Thus, the logistic regression was performed to ascertain the relation of education level (BA, MA, PhD), chapter affiliation (West, East, Atlantic, National Chapter), employer type (Public, Private), evaluation focus in job (primary, major, minor), and length of time practising evaluation (5 years or less, 6-15 years, 16 years or more) on the likelihood that CES members would hold the CE designation. Missing data fields resulted in a smaller sample size of $1,282(\mathrm{CE}=252$; non-CE $=1030)$ to facilitate working with complete data for each case. Dummy variables (binary variables) were created using the SPSS Categorical subcommand (SPSS v25) for the categorical predictor variables with more than two levels, and a reference category for comparison purposes was designated for each variable (education level-reference category designated as BA; chapter affiliation-reference category designated as Eastern Region; evaluation focus-reference category designated as minor focus; and time practising evaluation-reference category designated as 5 years or less). The results of the regression analysis with respect to each predictor were interpreted in relation to respective reference categories. Taken together, these steps in the logistic regression facilitated analyzing each CES member's pattern of responses on these socio-demographic variables and assessing the probability that he or she holds the CE designation. All of the predictor variables were entered simultaneously in one step, allowing for the unique contribution of each predictor (i.e., over and above that of all other predictors) to be assessed.

The initial regression model was statistically significant, $\chi^{2}(10)=252.38$, $p<0.001$, indicating that this set of socio-demographic characteristics was able 


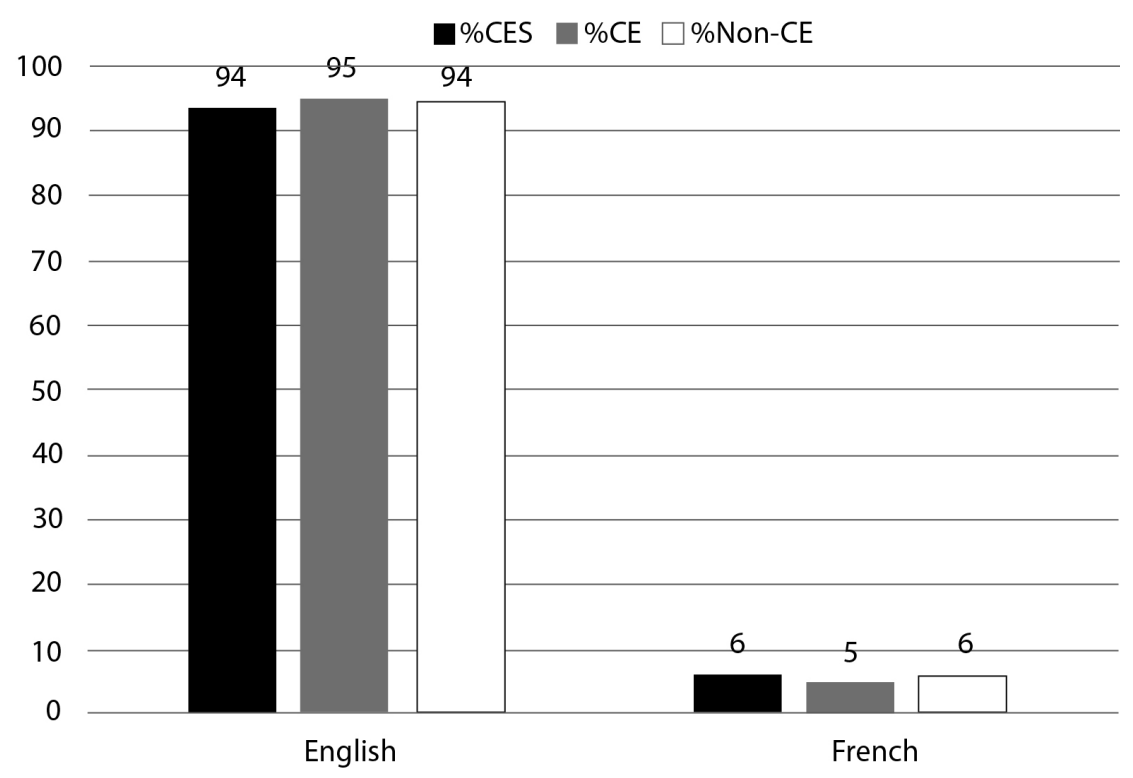

Figure 8. Preferred language: percentages by CE status

to distinguish between CES members with and without CE status. The model explained 28.4\% (Nagelkerke $R^{2}$ ) of the variance in CE designation and correctly classified $81.5 \%$ of cases. However, although the specificity (true negative rate) was impressive in that $96 \%$ of non-CE holders were correctly identified, the sensitivity of the test (true positive rate) was underwhelming, as only $22 \%$ of CE holders were correctly predicted. By default, when running a logistic regression, SPSS classifies cases using a cut-off probability of 0.5 (a case is assigned to the outcome category in question-in this case CE status-if the regression model predicts a probability of greater than 0.5 ). Lowering the cut-off value to 0.38 (the optimal value for balancing sensitivity and specificity determined by examining the outcomes of various classification points accompanying the receiver operating characteristic $[\mathrm{ROC}]$ analysis) resulted in a slightly lower overall classification rate of $80.4 \%$ and a reduction in correctly identifying those without the CE designation (89\%). However, this lower classification value did result in increasing the correct identification of CE holders to $45 \%$, which is a substantial improvement.

Table 2 shows the regression coefficients, Wald statistics, odd ratios, and $95 \%$ confidence interval for each of the predictors. An examination of the Wald statistics revealed that all five predictors, at the higher order level, significantly contributed to the prediction of CE status (all $p<0.05$ ). However, not all categories of the dummy variables reached statistical significance. The specific findings in relation to each predictor are described below.

The results indicate that type of employer is a significant predictor of CE status, $z=17.84, p<0.001$. An examination of the odds ratio reveals that CES 
Table 2. Logistic regression analysis of CE status as a function of demographic variables

\begin{tabular}{|c|c|c|c|c|c|c|}
\hline \multirow[b]{2}{*}{ Variables } & \multirow[b]{2}{*}{$B$} & \multirow[b]{2}{*}{ Wald test } & \multirow[b]{2}{*}{$p$ value } & \multirow{2}{*}{$\begin{array}{l}\text { Odds } \\
\text { ratio }\end{array}$} & \multicolumn{2}{|c|}{$\begin{array}{c}95 \% \mathrm{Cl} \text { for odds } \\
\text { ratio }\end{array}$} \\
\hline & & & & & Upper & Lower \\
\hline Employer & 0.69 & 17.84 & $<0.001$ & 2.00 & 1.45 & 2.77 \\
\hline Education & & 6.05 & 0.048 & & & \\
\hline PhD & 0.73 & 5.67 & 0.017 & 2.08 & 1.14 & 3.81 \\
\hline MA & 0.47 & 2.62 & 0.105 & 1.60 & 0.91 & 2.81 \\
\hline CES Chapter & & 11.01 & 0.012 & & & \\
\hline National & 0.48 & 5.14 & 0.023 & 1.62 & 1.07 & 2.46 \\
\hline West & 0.44 & 4.80 & 0.029 & 1.55 & 1.05 & 2.28 \\
\hline Atlantic & 0.93 & 8.69 & 0.003 & 2.53 & 1.37 & 4.69 \\
\hline Time in Evaluation & & 91.34 & $<0.001$ & & & \\
\hline Over 16 years & 2.61 & 88.34 & $<0.001$ & 13.56 & 7.87 & 23.35 \\
\hline 6-15 years & 1.76 & 42.80 & $<0.001$ & 5.83 & 3.44 & 9.90 \\
\hline Focus on Evaluation & & 24.97 & $<0.001$ & & & \\
\hline Primary & 1.13 & 18.67 & $<0.001$ & 3.09 & 1.85 & 5.16 \\
\hline Major & 0.48 & 3.28 & 0.070 & 1.63 & 0.96 & 2.75 \\
\hline (constant) & -4.88 & 126.51 & & & & \\
\hline
\end{tabular}

members employed within the private sector or who are self-employed are twice as likely to hold the CE designation as those who are employed within the public sector (i.e., employed within government or university settings). With respect to education level, only $\mathrm{PhD}$ status reliably predicted $\mathrm{CE}$ status, $z=5.67, p=0.017$. As the BA level was designated as the reference category, the odds ratio indicates that $\mathrm{CES}$ members with a $\mathrm{PhD}$ are twice as likely to have $\mathrm{CE}$ status as those with a BA.

CES Chapter affiliation, with the Eastern region designated as the reference category, emerged as an overall significant predictor within the regression equation, with each category of chapter affiliation predicting CE status. Specifically, compared to those affiliated with the Eastern Region chapters (Ontario, SQEP), those members associated with the Atlantic Chapter were two-and-a-half times more likely to hold the CE designation, $z=8.69, p=0.003$. Although the odds ratios were slightly lower in magnitude, members of the National Capital Chapter $(z=5.14, p=0.02)$ and the Western Chapter $(z=4.80, p=0.03)$ were also more likely to be CEs than those in the Eastern region (about 50\% more likely).

Length of time practising evaluation and evaluation focus in job were both positively associated with holding CE status. Specifically, those practising evaluation between 6 and 15 years were almost six times as likely to hold the CE credential compared to newcomers to field (those practising 5 years or less), while those members practicing for 16 years or more were over 13 times more likely to hold the designation than newcomers. Finally, only the category of major focus 
on evaluation within current employment reliably predicted CE status, with those within this category being three times more likely to possess the CE designation than those whose employment involves only a minor focus on evaluation.

\section{DISCUSSION AND CONCLUSIONS}

As of May 2017, just under 18\% of the CES membership held CE status, representing a marginal increase in uptake from the $16 \%$ reported in 2014 (Gauthier et al., 2015). With respect to the demographic profile, the findings from both the bivariate and multivariate analyses in the current examination were generally similar. The multivariate analysis we conducted facilitated sorting out the statistical influence of each predictor variable on the binary dependent variable in this analysis (CE status). When we examined the multivariate findings, a profile of CE holders emerged. Specifically, CES members who have earned the CE designation tend to be in the private sector, have a $\mathrm{PhD}$, be evaluators for a longer period of time, have evaluation as the major focus of their professional activities, and be from the Atlantic Region, the National Capital Chapter, or Western Canada. The reference categories for the variables in the logistic regression help to round out this profile of CES members in that those who are not likely to hold the CE designation tend to be younger (keep in mind that age is highly correlated with evaluation experience), have a Bachelor's Degree, be in the public sector, have evaluation as a minor focus of their professional practice, and be from Ontario (outside of the National Capital Region) or Quebec.

This profile of current CEs is similar to that outlined in the CES survey conducted in 2014 (Gauthier et al., 2015) and indicates relative stability in rates of CE uptake across demographic groupings within the CES membership that continues to the present. The CE designation is predominantly held by senior evaluators in the private sector practising outside of Ontario/Quebec (National Chapter region excluded). There are numerous contextual factors that may account for such differential uptake rates among the membership. Rational choice models of behaviour (e.g., Ajzen \& Fishbein, 1980) posit that people are logical decision-makers and will weigh the options before them and choose the course of action that maximizes benefits and minimizes risks. Thus, the optimal (or most rational) choice is the one that promises to provide the decision-maker with the greatest benefits and usefulness, and therefore the greatest level of post-decisional satisfaction. The decision to seek the CE designation can be framed and critically examined within this rational choice perspective, and the associated costs and benefits may explain the CE demographic profile.

Individuals contemplating the $\mathrm{CE}$ designation would first examine the potential benefits, which include recognition of professional competence along with enhanced professional status, which can bestow a competitive edge in the field (Gauthier et al., 2015). In line with the principles of social identity theory (Tajfel \& Turner, 2004), the designation can also be perceived as a means of establishing or strengthening one's identity as a member of the evaluation community, with 
CE holders more likely than non-holders to feel part of a larger profession and to follow CES professional practice guidelines in their practice (Gauthier et al., 2015). It is very likely that these aspects of the CE designation would be viewed as more beneficial to certain demographic groupings of evaluators than others. Specifically, those whose workplace requires recognized status and/or a competitive edge, and those who highly value the professional identity of evaluation would be most likely to place emphasis on these benefits. Consultants in the private sector are likely to perceive the most benefit from obtaining a competitive edge in the evaluation-practice field. In contrast, many in the public sector, especially federal government employees, report that their employer does not view the CE designation as credible or useful (Gauthier et al., 2015), thus eliminating this potential benefit and contributing to differential uptake rates across both private and selfemployment fields and certain regions. Further, it is likely that individuals who have focused their career on evaluation for longer periods of time are more likely to professionally identify as an evaluator and seek ways to strengthen and solidify that identity, even in the absence of instrumental benefits (Tajfel \& Turner, 2004). Taken together, it is most likely that older, more experienced practitioners and private-sector evaluators would be the groupings to perceive the most benefit from the designation.

Within the rational choice framework, the associated costs of seeking the designation would also be considered by prospective applicants. The most substantial pertinent costs are the time and effort required to complete what is perceived as a difficult application package, and the financial cost in the form of the application fee. In fact, when surveyed, just over half of CES members who had chosen not to pursue the designation indicated that the time demands and the expense associated with the application were important factors in their decision (Gauthier et al., 2015). Highlighting the relevance of a cost-benefit analysis framework, almost three-quarters of these individuals also indicated that they perceived insufficient benefit to obtaining the credential. The modest and demographically divergent uptake rate of the credential to date is not surprising, given the immediate and significant costs associated with the application process and the uncertain tangible benefits for many members.

Beyond the differential valuing of the costs and rewards associated with the designation, there is another possible explanation that might contribute to the demographic profile of CE holders-specifically, the honorary CE status (to become Credentialing Board members) and the fast-track CE application option that was offered for one year to long-time CES members who had more evaluation experience (both of these factors are conflated with age) and reported that evaluation was the major focus of their employment. Given that these individuals were specifically targeted for a CE process that minimized costs, it is likely that part of the current CE roster is composed of these older, more experienced evaluators who focus primarily on evaluation within their employment (Gauthier et al., 2015). Unfortunately, within our database we could not distinguish between those who received the designation as an honorary status versus the fast-track process 
versus the standard application route, so the extent to which this underlies the demographic profile is not known.

Regardless, the current statistical analysis suggests overall patterns. One way to interpret these patterns is to examine the de facto interpretation of the criteria that are used by CES to review CE applicants. The process of ascertaining competence (judged by members of the Review Board based on written documentation submitted by CE applicants) involves applicants self-reporting the extent to which their knowledge and experience (reflected in their education and practice) have resulted in their being able to claim competence in each of the sub-criteria (total of 49) in the five competency domains (reflective practice, technical practice, situational practice, management practice, and interpersonal practice) (CES, 2010).

The profile of successful CE applicants indicates that more experienced (older), more highly educated $(\mathrm{PhD})$, private-sector, full-time evaluators tend to be CEs. Conversely, younger, less experienced, less well-educated, part-time evaluators in CES are less likely to have the CE designation. These profiles suggest implications for the sustainability of the CE designation into the future. Right now, the CE designation is disproportionately held by evaluators who are well into their careers and are highly educated. Arguably, the value of securing a CE for their careers is less than for younger, less experienced, and less well-educated evaluators who are beginning their careers. The current pattern of who is successful in securing the $\mathrm{CE}$ designation suggests the possibility that in the future, the reach of the CE designation will be self-limiting. That is, it does not tend to attract CES members who stand to benefit from it the most, likely due to a combination of uncertainty regarding the actual benefits, the immediate substantial costs associated with the application process, and the application criteria. Following the logic inherent in the rational choice model, the implications are clear: To increase CE uptake rate among the membership, the benefits of the designation must be clear and relevant to those sectors of the membership that are currently unconvinced, and/or the costs must be reduced. Assuming that it is infeasible to reduce the associated costs, a focus on amplifying the benefits of CE status would be the most productive route.

In their evaluation of the PDP, Fierro et al.s (2016) first recommendation focused on ways to increase the value of the $\mathrm{CE}$ designation among consumers of evaluation services. In that recommendation, Fierro et al. pointed out that the $\mathrm{CE}$ designation is "broad and generic" ( $p$ 54):

Interviewees often suggested that tiers or specializations could enhance the value of the CE designation. For example, this might include creating tiers that indicate the level of expertise (e.g., beginner, intermediate, advanced, expert), differentiating between those who manage versus implement evaluations, and offering specializations based upon type of evaluation approach. (p. 54)

Although this recommendation is intended to increase the value of the CE among consumers of evaluations, it has implications for the suppliers of evaluation services. Offering $\mathrm{CE}$ designation options that recognize both the experience levels 
of applicants and the diverse nature of our profession could make the CE more attractive to prospective applicants. Encouraging younger evaluators (perhaps by emphasizing more the importance of graduate education specializing in program evaluation) to apply for and secure the CE designation could benefit both individual evaluators and the Canadian Evaluation Society.

This analysis has reported on the demographic characteristics that distinguish CES members that hold the CE designation from the rest of the CES member body. One issue that should be kept in mind when interpreting these findings is the large amount of missing data in the CES membership database. The sociodemographic information contained in the database is self-populated by members on their member profile, and, as documented in Table 1, a substantial number of members are opting to not fully complete these profiles. These missing data points resulted in only approximately three-quarters of the membership being included in the regression analysis. In fact, almost $20 \%$ of CEs in particular were omitted from the regression analysis due to missing data. Although it was beyond the scope of the current analyses, it is possible that non-random patterns within the missing data (e.g., differential tendencies of sub-groups of members within the membership base to supply personal information) might affect the findings. This potential limitation in the available data, however, also represents valuable information for future research. Encouraging all CES members to provide basic sociodemographic information would facilitate future efforts to monitor trends in the uptake of the designation and to adequately evaluate the CE designation program.

The data limitations notwithstanding, this paper provides a clear picture of the prototypical CE holder as a mature, highly educated, and experienced evaluator. As other national evaluation associations move toward offering their members ways of distinguishing themselves professionally (Julnes \& Bustelo, 2017), the Canadian Evaluation Society should note these findings and take advantage of its current leadership position to advance professionalization opportunities for all of its members.

\section{REFERENCES}

Adrien, M., \& Hellrung, L. (2019). The journey to a professional designation for evaluators in Canada. In J. Furubo \& N. Stame (Eds.), The evaluation enterprise: A critical view (pp. 198-220). New York, NY: Routledge.

Ajzen, I., \& Fishbein, M. (1980). Understanding attitudes and social behavior. New York, NY: Springer Verlag.

Altschuld, J. W. (2005). Certification, credentialing, licensure, competencies, and the like: Issues confronting the field of evaluation. Canadian Journal of Program Evaluation, 20(2), 157-168.

Altschuld, J. W., \& Engle, M. (2015a). Editors' notes. New Directions for Evaluation, 145, 1-4. https://doi.org/10.1002/ev.20107

Altschuld, J. W., \& Engle, M. (2015b). The inexorable historical press of the developing evaluation profession. New Directions for Evaluation, 145, 5-19. https://doi.org/10.1002/ev.20108 
Buchanan, H. (2015). A made-in-Canada credential: Developing an evaluation professional designation. Canadian Journal of Program Evaluation, 29(3), 33-53. https:// doi.org/10.3138/cjpe.29.3.33

Buchanan, H., \& Kuji-Shikatani, K. (2013). Evaluator competencies: The Canadian experience. Canadian Journal of Program Evaluation, 28(3), 29-47.

Canadian Evaluation Society (CES). (n.d. a). For candidates: Why should you do it? Retrieved from https://evaluationcanada.ca/candidates

Canadian Evaluation Society (CES). (n.d. b). For credentialed evaluators. Retrieved from https://evaluationcanada.ca/credentialed-evaluators

Canadian Evaluation Society (CES). (2010). Competencies for Canadian evaluation practice. Retrieved from http://evaluationcanada.ca/txt/2_competencies_cdn_evaluation_ practice.pdf

Castro, M. P., Fragapane, S., \& Rinaldi, F. M. (2016). Professionalization and evaluation: A European analysis in the digital era. Evaluation, 22(4), 489-507. https://doi. org/10.1177/1356389016667887

Fierro, L. A., Galport, N., Hunt, A., Codd, H., \& Donaldson, S. I. (2016). Canadian evaluation society credentialed evaluator designation program. Claremont, CA: Claremont Evaluation Center.

Furubo, J., \& Stame, N. (2019). The evaluation enterprise: A critical view. New York, NY: Routledge.

Gauthier, B., Kishchuk, N., Borys, S., \& Roy, S. N. (2015). The CES professional designations program: Views from members. Canadian Journal of Program Evaluation, 29(3), 98-133. https://doi.org/10.3138/cjpe.29.3.98

Gussman, T. (2005). Improving the professionalization of evaluation. Prepared for the Centre of Excellence in Evaluation. Ottawa, ON: Treasury Board of Canada Secretariat. Retrieved from https://www.tbs-sct.gc.ca/cee/prof-eval-eng.asp

Halpern, G., Gauthier, B., \& McDavid, J. C. (2015). Professional standards for evaluators: The development of an action plan for the Canadian Evaluation Society. Canadian Journal of Program Evaluation, 29(3), 21-32. https://doi.org/10.3138/cjpe.29.3.21

Howell, D. C. (2010). Fundamental statistics for the behavioral sciences (7th ed.). Belmont, CA: Wadsworth Publishing.

Jacob, S., \& Boisvert, Y. (2010). To be or not to be a profession: Pros, cons and challenges for evaluation. Evaluation, 16(4), 349-369. https://doi.org/10.1177/1356389010380001

Julnes, G., \& Bustelo, M. (2017). Professional evaluation in the public interest(s). American Journal of Evaluation, 38(4), 540-545. https://doi.org/10.1177/1098214017729876

Kuji-Shikatani, K. (2015). Credentialed evaluator designation program: The Canadian experience. New Directions for Evaluation, 145, 71-85. https://doi.org/10.1002/ev.20112

Kuji-Shikatani, K., Thompson, M., \& Matthew, M. (2015). Launching the Credentialed Evaluator (CE) designation. Canadian Journal of Program Evaluation, 29(3), 70-85. https://doi.org/10.3138/cjpe.29.3.70

Long, B. (2006). Minority report with respect to professional designations for evaluators. Retrieved from http://www.evaluationcanada.ca/txt/5_minority.pdf 
Love, A. (1994). Should evaluators be certified? New Directions in Program Evaluation, $62,29-40$.

Love, A. (2015). Building the foundation for the CES professional designation program. $\mathrm{Ca}$ nadian Journal of Program Evaluation, 29(3), 1-20. https://doi.org/10.3138/cjpe.29.3.1

Mason, S., \& Hunt, A. (2018). So what do you do? Exploring evaluator descriptions of their work. American Journal of Evaluation, 40(3), 395-413. https://doi. org/10.1177/1098214018767049

McDavid, J. C., \& Huse, I. (2015). How does accreditation fit into the picture? New Directions for Evaluation, 145, 53-69. https://doi.org/10.1002/ev.20111

Montrosse-Moorhead, B., Bellara, A., \& Gambino, A. J. (2017). Communicating about evaluation: A conceptual model and case example. Journal of MultiDisciplinary Evaluation, 13(29), 16-30.

Picciotto, R. (2011). The logic of evaluation professionalism. Evaluation, 17(2), 165-180. https://doi.org/10.1177/1356389011403362

Schwandt, T. A. (2015). Evaluation foundations revisited: Cultivating a life of the mind for practice. Stanford, CA: Stanford University.

Tabachnik, B. G., \& Fidell, L. S. (2013). Using multivariate statistics (6th ed.). Boston, MA: Pearson Education.

Tajfel, H., \& Turner, J. C. (2004). The social identity theory of intergroup behaviour. In J. T. Jost \& J. Sidanius (Eds.), Political psychology: Key readings in social psychology (pp. 276-293). New York, NY: Psychology Press.

\section{AUTHOR INFORMATION}

Karen Lawson is a professor of psychology at the University of Saskatchewan and the current chair of the Consortium of Universities for Evaluation Education (CUEE). Trained as an applied social psychologist, she focuses on conducting community-based program evaluation research. She teaches program evaluation at the graduate level and has supervised over 30 program evaluation-related student internships in the community.

Theresa Hunter is an independent consultant in Victoria, British Columbia. Her research interests include children's rights, quality child care, Aboriginal Head Start, research ethics, and graduate evaluation education. Her publications include articles on quality child care, a 2018 inventory of Canadian graduate evaluation education programs, and a recent book chapter on children's rights in Canada.

Jim McDavid is prefessor Emeritus in the School of Public Administration at the University of Victoria. His research covers program evaluation, performance measurement, and performance management in both Canada and the United States. His recent publications focus on teaching evaluation and professionalizing the field. The third edition of his textbook, Program Evaluation and Performance Measurement: An Introduction to Practice, was published by Sage Publications in 2019. 